


\section{Exercise testing: why, which and how to interpret}

\section{Educational aims}

To address some questions that often arise when exercise testing is performed in the clinical routine:

Dhat are the indications to perform an exercise test?

Which test should be used for which situation?

How should the test be carried out in practice?

How should the results of the test be interpreted?

\section{Summary}

Exercise testing is widely applied in clinical practice. The aim of this article is to address some questions that often arise when exercise testing is performed in clinical routine. Two complementary tests will be discussed in more detail: the maximal incremental ergometer test and the timed walk test.

First, the indication to perform exercise testing is discussed. The second question is which test to apply and how to carry out the test in practice. Finally, the interpretation of the results of the tests are discussed.

\section{Is exercise testing useful?}

When deciding to carry out an exercise test, the reason(s) for doing it should be specified and, in addition, the appropriate type of exercise test and protocol should be chosen.

Exercise tests are performed in patients in order to establish whether exercise capacity is impaired, to investigate the underlying cause of the limitation and to establish the risks of exercise. In addition, dyspnoea during exercise is a frequent reason to perform an exercise test. Evidence suggests that symptoms such as dyspnoea, impaired exercise tolerance and reduced quality of life, common experiences in patients with chronic respiratory disease, have only a weak correlation with lung function impairment (figure 1) [1], and that prediction of exercise performance based solely on resting pulmonary function tests is inaccurate [3-5].

Exercise testing can be performed in order to obtain a specific diagnosis (e.g. exercise-

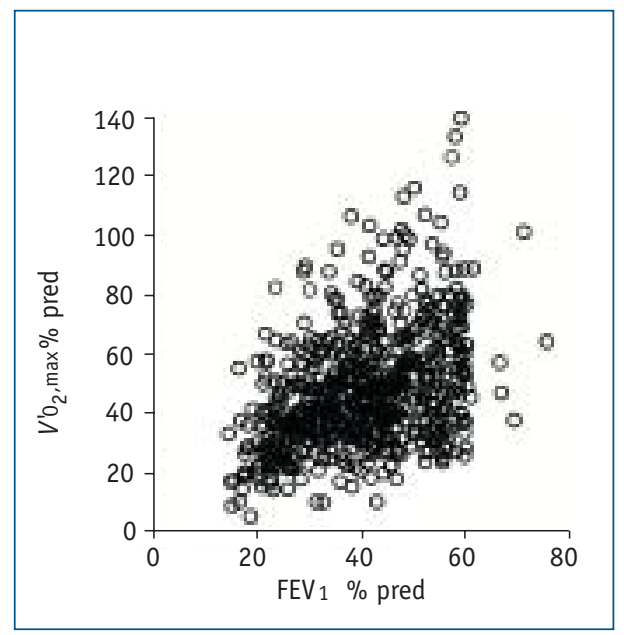

R. Gosselink

T. Troosters

M. Decramer

Respiratory Rehabilitation and Respiratory Division, University Hospitals, and Faculty of Physical Education and Physiotherapy, Katholieke Universiteit Leuven, Leuven, Belgium.

Correspondence:

R. Gosselink

Professor of Respiratory

Rehabilitation

Respiratory Rehabilitation

Division

University Hospital Gasthuisberg Herestraat 49

B-3000 Leuven

Belgium

Fax: 3216346866

E-mail:

rik.gosselink@uz.kuleuven.ac.be

Figure 1

Relationship between pulmonary function (forced expiratory volume in one second (FEV 1$)$ ) and exercise capacity (maximal oxygen uptake $\left(\mathrm{V}^{\prime} \mathrm{O}_{2}\right.$, max $)$ ) in patients with chronic obstructive pulmonary disease. Figure modified from [2]. 
induced asthma), to answer particular questions concerning working capacity (employment) or to a predict a level of risk (i.e. operability for lung resection, survival). In many diseases, including chronic obstructive pulmonary disease (COPD), primary pulmonary hypertension and cystic fibrosis, exercise tolerance has been shown to be one of the most important predictors of mortality or morbidity. Finally, exercise testing can be used to quantify objective gains after interventions, for example after medication, surgical procedures or rehabilitation.

If a specific question is being asked, clinicians may use complex exercise tests that can accurately measure pulmonary gas exchange and aspects of the cardiocirculatory and muscular systems, or they may prefer more simple, yet useful, tests to answer clinical questions. Maximal incremental exercise tests may be required in the first case, whereas field walking tests may suffice in the latter.

\section{Which tests are available and how should the appropriate test be chosen?}

\section{Incremental exercise testing}

The gold standard in exercise testing is the incremental exercise test. Incremental exercise testing is the first choice for the following: assessing impaired exercise capacity, investigating factors limiting exercise performance, or assessing the risks of participating in exercise programmes or prescribing exercise training. For all these indications, incremental exercise testing is needed to provide clinicians with key data that cannot be obtained from resting measures of pulmonary function, cardiac function, blood gases or other exercise tests. The introduction of computerised breath-by-breath equipment has made incremental exercise testing available in most clinical settings [6], and, subsequently, standardised maximal exercise tests have been developed [7-9]. In addition, the estimation of peak exercise responses based upon submaximal exercise data (mainly heart rate (HR)) is inappropriate in COPD patients, since these patients often do not reach their maximal $H R$.

Two types of ergometer can be used for incremental exercise testing: a treadmill or a cycle ergometer. When a motor-driven treadmill is used, increasing the speed and/or inclination imposes the external workload, and this workload is dependent on body weight. Factors such as walking efficiency (depending on footwear, length of the lower limb and training status on treadmills) and the use of arm support may have an unpredictable influence on the oxygen uptake $\left(\mathrm{VO}_{2}\right)$ profile during treadmill testing. However, compared with cycling, walking is a more natural movement and less lactate is produced at iso$\mathrm{VO}_{2}$. Treadmill walking is generally advised for paediatric exercise testing. When a cycle ergometer is used, the workload is better controlled and the external work needed is less dependent on body weight as compared with the treadmill. The unloaded cycling $\mathrm{V}^{\prime} \mathrm{O}_{2}$ is dependent on the weight of the lower limbs; however, when a load is added, the $\mathrm{VO}_{2}$ will increase further, independent of body weight $\left(\approx 10 \mathrm{~mL} \cdot \mathrm{min}^{-1} \cdot \mathrm{W}^{-1}\right)$. In addition, cycle ergometry, unlike treadmill ergometry, allows the investigator to compare patients' responses to iso-submaximal work and permits a more clear insight into mechanical efficiency. The stability of the patient on a bicycle results in less noise on electrocardiography (ECG) and blood pressure recordings. Arterial and venous catheters can also be accessed more easily on a cycle ergometer when compared with a treadmill. If a cycle ergometer is going to be used for exercise testing, it should be electromagnetically braked and the workload should be adjustable in steps of 5-10 W.

\section{Field tests}

Field tests, such as the 6- or 12-minute walk test (6- or 12MWT), shuttle walk test (SWT) or constant workload endurance test, are used to investigate the effects of interventions that alter submaximal or endurance exercise capacity, for example in respiratory rehabilitation [6]. The outcome of these walk tests is able to more closely mimic the functional abilities needed in everyday life [10]. It has been reported that field tests are performed in $\sim 80 \%$ of the rehabilitation programmes in the USA [11]. Although patients are free to change their speed during a timed walk test, they generally maintain a very stable speed [12], which results in a steady-state $\mathrm{VO}_{2}$ and a walking speed that represents the critical walking speed (i.e. the speed that can be maintained) [13]. This property may make the test sensitive to changes due to interventions improving the critical power (i.e. exercise training). 


\section{A combination}

Incremental exercise testing and field testing have complementary value in areas such as pulmonary rehabilitation [14]. Prior to participation in a respiratory rehabilitation programme, maximal exercise performance has to be established for the diagnosis of impaired exercise capacity to be made, the identification of factors limiting exercise performance and the prescription of exercise training. However, during follow-up, valuable clinical information can be obtained from constant work rate tests or walk tests. Field exercise tests, such as the 6- or 12MWT [3], the shuttle run test, the SWT [15] and the step test, are easy to perform and are valid, as they are more related to activities of daily living [16]. For some of these tests, however, lack of reference values and the absence of physiological measures are important limitations. In healthy subjects, a fairly good correlation with maximal $\mathrm{VO}_{2}\left(\mathrm{VO}_{2}\right.$, $\left.\mathrm{max}\right)$ is observed, although it is not good enough to predict $\mathrm{V}_{2}, \max$ in individual subjects [17-19].

\section{Incremental exercise testing}

An incremental exercise test consists of baseline measurements lasting at least $2-3$ minutes, then a warm-up period of 3 minutes unloaded work, followed by the incremental part of the exercise test. The choice of an adequate increment size is one of the important steps in tailoring a test to each individual patient. Ideally, peak work rate should be reached within 8-12 minutes. Work rate increments have important consequences when the exercise results are to be used for exercise prescription in a pulmonary rehabilitation programme. Workload increment size does not affect $\mathrm{VO}_{2}$, max, ventilation or $\mathrm{HR}$, but results in significant differences in maximal workload [20]:

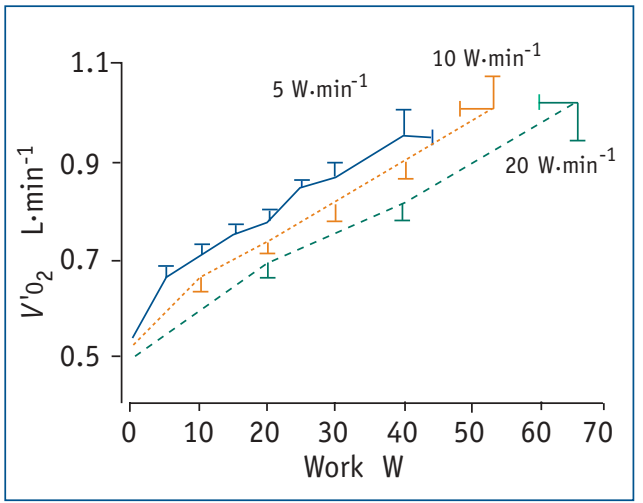

protocols with larger increments result in a higher peak workload (figure 2). Consequently, this may impact on the exercise prescription, as it is frequently based on peak work rate.

After peak exercise is reached, patients should continue cycling at very low work rate levels to avoid sudden drops in blood pressure due to pooling of venous blood. When patients are tested consecutively, the increment size should be kept identical.

\section{Measurements}

In clinical practice, the measurements taken include the following: work rate, 12-lead ECG, blood pressure, pulmonary gas exchange $\left(\mathrm{VO}_{2}\right.$ and carbon dioxide production $\left.\left(\mathrm{VCO}_{2}\right)\right)$, ventilation, transcutaneous oxygen saturation and symptom scores. When arterial blood gases are available at rest and peak exercise, most clinical problems regarding exercise limitation can be addressed. Standardisation and technical procedures for reproducible exercise testing have been described in a recent American Thoracic Society (ATS)-American College of Chest Physicians (ACCP) document [6]. Over the last 15 years it has become more routine to have specially trained non-physicians (physiologists, physiotherapists and technicians) conducting exercise tests, with a consultant immediately avalible for consulation or emergency situations [21]. Symptom scores for dyspnoea and exertion have also been shown to be valuable tools during exercise testing. Visual analogue scales and Borg scales cannot be interchanged, but both show good agreement and, hence, are valid tools for evaluating symptoms of dyspnoea and perceived exertion [22]. Variables at submaximal exercise and at peak exercise are integrated to answer questions on exercise capacity, safety of exercise and limitation of exercise, and specific questions, such as exercise prescription.

\section{How to interpret the maximal exercise test}

The main outcome of the maximal exercise test is $\mathrm{V}^{\prime} \mathrm{O}_{2}$, max , standardised per kilogram of body weight. Alternatively, comparison with reference values allows the level of exercise impairment to be judged. The European Respiratory Society (ERS) report on exercise impairment in patients with respiratory disease [23] describes two equations used as reference values for $\mathrm{VO}_{2}$, max. The equation of Jones et al. [24] seems useful in clinical practice for cycle ergometry (table 1). Exercise laboratories should be encouraged to
Figure 2

Exercise protocols for incremental exercise testing. Reproduced with permission from [20]. 


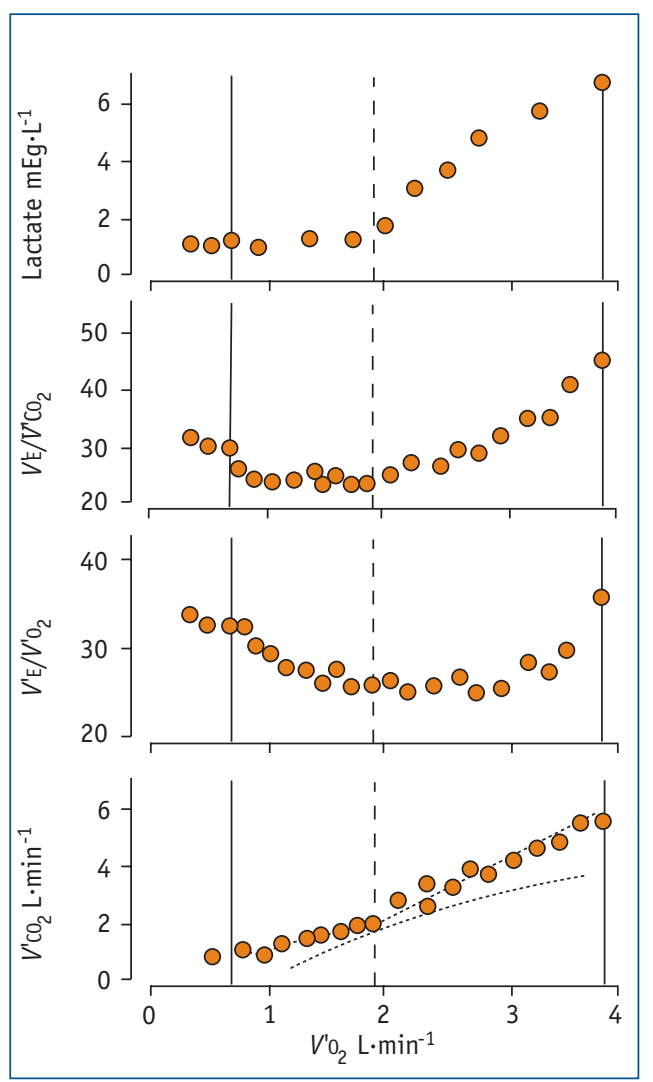

$( \pm 10)$ beats.minute $\left.{ }^{-1}\right)$ is indicative of maximum cardiac output. This limitation is observed in healthy subjects and, frequently, in patients with a forced expiratory volume in one second ( $\mathrm{FEV}_{1}$ ) $>50 \%$ predicted [32]. This exercise limitation is nota direct consequence of pulmonary disease. In other conditions, such as heart failure or ischaemia, the HRmax may not be reached, but a low ratio of $\mathrm{VO}_{2} / \mathrm{HR}$ (oxygen puls) and a high submaximal $H R$, together with other findings (echocardiography, ECG changes), may help to identify cardiocirculatory limits.

A ventilatory limitation is related to an imbalance between load and capacity of the respiratory muscles (pump). The load on the pump can be increased due to airway obstruction, altered lung/chest wall compliance and hyperinflation. In addition to altered lung mechanics, the capacity of the ventilatory pump might be impaired due to respiratory muscle weakness [33] and/or reduced ventilatory drive. This might be indicated as a decrease in the slope of $V^{\prime} \mathrm{E}$ to $\mathrm{V}^{\prime} \mathrm{CO}_{2}$ or by an increase in end-tidal carbon dioxide. The increased ratio of the pressure needed and the pressure that can be maximally generated indicates the risk for potential respiratory muscle failure.

A ventilatory limitation is frequently observed in patients with more advanced lung disease, airway obstruction, chest wall deformities, respiratory muscle disease/weakness and in patients with interstitial lung disease. A ventilatory limitation can be identified in different ways. First, an increase in arterial $\mathrm{CO}_{2}$ tension $\left(\mathrm{Pa}_{1} \mathrm{CO}_{2}\right)$ can be observed during the exercise test, but this response is also influenced by central drive. Secondly, V'E that exceeds $70-80 \%$ of the maximal voluntary ventilation (MW; often calculated as $\sim 37.5 \times \mathrm{FEV} 1$ ) is considered a ventilatory limitation. More recently, the assessment of dynamic hyperinflation during exercise (reducing the capacity of the ventilatory pump) by measurements of inspiratory capacity or flow-volume loops has been found to be useful [34].

Pulmonary gas exchange limitation is identified by an isolated reduction in arterial oxygen tension $\left(\mathrm{Pa}_{1} \mathrm{O}_{2}\right)$ and/or an increase in the alveolar-arterial oxygen gradient $\left(D \mathrm{~A}-\mathrm{a}_{1} \mathrm{O}_{2}\right)$ of $>2 \mathrm{kPa}$ [25]. It is often unclear why patients with pulmonary gas limitation stop exercising; some do not sense hypoxaemia and continue to exercise up to very low values of $\mathrm{Pa}_{2} \mathrm{O}_{2}$, while other patients stop exercise when $\mathrm{Pa}_{1} \mathrm{O}_{2}$ has hardly decreased [35]. The maximal exercise test will be terminated by the investigator when oxygen saturation drops below $80 \%$. A low transfer factor of the lung for carbon monoxide $(T L, C O)$ has some prediction for exercise-induced hypoxaemia [36]. In the absence of significant airflow limitation, $T L, C O$ values $<50 \%$ predicted will be associated with hypoxaemia in most patients. In contrast, in patients with more severe COPD (and loss of diffusion capacity), ventilation-perfusion inhomogeneity might decrease during exercise and the net effect will be that $\mathrm{Pa}_{\mathrm{O}_{2}}$ and $\mathrm{DA}_{\mathrm{A}}-\mathrm{a}_{1} \mathrm{O}_{2}$ do not alter [37].

Peripheral muscle weakness may also contribute to reduced exercise capacity [38-40]. In this case, at the end of the exercise test, patients will rate their level of exertion as high, using the Borg score. Muscle strength measurements of leg and arm muscles are needed to further explore muscle weakness as the limiting factor of exercise performance. Peripheral gas exchange problems are more difficult to address. In routine clinical testing, complaints of claudicate pain may be suggestive of peripheral gas exchange limitation.

Finally, psychological factors, such as fear, anxiety or lack of motivation, can also contribute to low exercise performance. A psychological limitation is diagnosed when no other limitations of exercise performance (as listed previously) can be identified and the behaviour of the patient would suggest it.
Figure 3

Lactate, $V^{\prime} E, V^{\prime} \mathrm{CO}_{2}, V^{\prime} E, V^{\prime} \mathrm{O}_{2}$ response and $V$-slope plot during an incremental maximal exercise test. Figure modified from [29]. 


\section{Field tests and endurance tests}

\section{Six or twelve minute walk test}

In the 6- or 12MWT, functional exercise capacity is expressed as the distance in meters covered by the patient in 6 or 12 minutes, respectively $[2,41]$. The test is performed in a corridor with a known track length. Patients are instructed to cover as many meters as possible in the time scheduled. If needed, patients are allowed to have rest periods during the test, and they are also allowed to restart or change the walking speed. At the end of the test, patients must have the feeling that the covered distance was the maximal that could be achieved. In addition to the distance covered, HR, transcutaneous oxygen saturation $\left(\mathrm{Stc}_{1} \mathrm{O}_{2}\right)$, and Borg ratings for dyspnoea and fatigue can easily be obtained at the end of the walking test. All these measures provide further information about the patient [42].

Encouragement during the test is standardised, and the test has to be repeated at least once [41]. It has been shown previously that further repetitions of the test produce only a limited improvement in the result $(\sim 3 \%)$. A recent guideline published by the ATS [43] and results obtained from the National Emphysema Treatment Trial [44] have both stressed the importance of proper standardisation. Indeed, appropriate standardisation resulted in a low coefficient of variation $(\approx 8 \%)$ [43]. To ensure reproducible and reliable results, the guidelines in table 3 have been proposed.

\section{Shuttle walk test}

The SWT is an incremental, externally paced exercise test that stresses the patient to a symptom-limited maximum [15]. The patient walks around a 9-m course, defined by two marker cones (total distance covered per shuttle is 10

Table 3 Guidelines for reproducible and reliable results in timed walk tests

- Prepare written instructions for all staff members involved, including spoken instruction given to the patient prior to the test

- Standardise the encouragement given, in terms of phrases used and timing; every 30 seconds, encouragement can be given to maximise patient effort

- Record oxygen saturation, heart rate and symptoms; use the validated Borg score to assess symptoms of dyspnoea and fatigue

- Supplementary oxygen should be provided during the test when necessary; the investigator should carry the oxygen cylinder

- Repeat the test at least once after a sufficient rest period (15-30 minutes) $\mathrm{m})$. The speed of walking is dictated by signals played from a tape cassette and increases every minute until the patient is unable to keep up with the speed due to breathlessness or fatigue. Hence, the $\mathrm{VO}_{2}$ profile during an incremental SWT mimics that of the maximal incremental tests [45]. The walking distance correlates fairly well with $\mathrm{VO}_{2}$, $\max [46]$, but, to date, no reference values are available. As this is a maximal incremental exercise test, the results obtained may overlap with those from an incremental exercise test. However, since no physiological measurements other than $\mathrm{HR}$ and $\mathrm{Stc}, \mathrm{O}_{2}$ are performed, the test does not allow interpretation of the cause of exercise limitation.

Oxygen desaturation, hypercapnia, $\mathrm{VO}_{2}$ and HR during field tests such as the 6MWT and the SWT are close to those obtained during incremental exercise testing [12]. Therefore, investigators should be trained in detecting alarm symptoms that may occur during this field exercise test (including pallor, angina, dizziness, extreme dyspnoea) and in basic life-saving procedures.

\section{Endurance shuttle walk test}

The endurance SWT is based on the SWT. The patient is asked to sustain walking as long as possible at a speed equal to $85 \%$ of the maximal walking speed during the SWT (after a practice walk) [47]. Walking speed during the endurance SWT is dictated by signals played from a tape cassette. The test is terminated when the patient is, for the second consecutive lap, unable to reach the cone in the time allowed. Good reproducibility is obtained after one practice test. The endurance SWT appears to be more sensitive to changes due to respiratory rehabilitation than the SWT [47].

\section{Cycle endurance test}

It has been shown that constant work rate tests performed on a cycle or treadmill are sensitive when following-up patients after respiratory rehabilitation and medication $[48,49]$. In addition, test-retest reliability is good [50]. These tests, however, require at least one maximal incremental exercise test preceding the consecutive follow-up tests. The follow-up tests are made at a fixed work rate (mostly $75 \%$ of the peak work rate of the preceding incremental test). Nevertheless, the data obtained during a constant work rate test are of physiological relevance. These tests can vary in complexity from analysis of exercise time, ventilation, $H R$ and symptom scores to the 
analysis of the organ system responses at the onset of exercise. $\mathrm{VO}_{2}, V^{\prime} \mathrm{CO}_{2}$, ventilation and $\mathrm{HR}$ responses are characterised by a time constant. This is the time needed to reach $63 \%$ of the final steady-state change [29]. This last approach is mostly reserved for research, and standardisation is critical to obtain reliable results. The power output that can be sustained (theoretically) forever is called the critical power. No data are available on normal values, but the critical power in normal subjects is $\sim 65 \%$ of the peak workload, whereas, in COPD patients, it was found to be slightly higher [51].

\section{How to interpret field tests}

The main outcome of a field test for the assessment of exercise capacity is the distance walked or the change in distance. The walking distance in the SWT correlates well with $\mathrm{V}_{2}$, $\max$ [46]. The availability of normal values for the 6MWT has contributed to the interpretability of the data from this test (table 4) [52, 53]. A walking distance $<82 \%$ predicted is considered abnormal [53]. No reference values are available for other field exercise tests. Most of these field tests are particularly important when evaluating changes over time. The interpretation of the clinical significance of changes (minimal clinically relevant

\section{Table 4 Reference values for the encouraged [53] and unencouraged [52] 6-minute walk test}

\author{
Male \\ Female \\ Height in $\mathrm{cm}$, weight in $\mathrm{kg}$, age in years. \\ difference (MCID)) is available for the 6MWT (an \\ MCID is a change of $54 \mathrm{~m}$ in the $6 \mathrm{MWT}$ [54]). This \\ has also contributed to the interpretability of data \\ from the 6MWT.
}

Encouraged: $218+(5.14 \times$ height $)-(5.32 \times$ age $)-(1.80 \times$ weight $)+51.31 \mathrm{~m}$

Unencouraged: (7.57 $\times$ height $)-(5.02 \times$ age $)-(1.76 \times$ weight $)-309 \mathrm{~m}$

Encouraged: $218+(5.14 \times$ height $)-(5.32 \times$ age $)-(1.80 \times$ weight $) \mathrm{m}$

Unencouraged: (2.11 $x$ height) $-(2.29 \times$ weight $)-(5.78 \times$ age $)+667 \mathrm{~m}$

\section{Conclusion}

In conclusion, exercise testing has gained popularity in a variety of clinical conditions and specifically in respiratory rehabilitation. Maximal incremental exercise testing has its main emphasis on the diagnosis of exercise impairment and the mechanisms related to this impairment. Field tests are most important in the longitudinal assessment of exercise performance, such as in the evaluation of treatment.

\section{Educational questions (Answers on page 129)}

1. What are the main indications for exercise testing?

2. When would you choose a maximal incremental test and when would you chose a field test in the evaluation of exercise performance?

3. Is the size of the increments of the work load in a maximal exercise test of importance to the outcome of the test?

4. Which guidelines have to be followed to ensure reliable field testing?

5. How is "ventilatory limitation" as a cause of exercise limitation defined?

6. Should the 6MWT be considered a submaximal test?

7. Can patients with COPD be limited in their exercise performance by reaching the boundaries of the cardiocirculatory system?

8. Is a maximal incremental test the test of choice to assess the effects of pulmonary rehabilitation?

9. Are measurements of peripheral muscle strength complementary to the information obtained from a maximal incremental exercise test?

10. Which reference value for $\mathrm{V}^{\prime} \mathrm{O}_{2}$, max should be used? 


\section{Suggested further reading}

\section{American Thoracic}

Society/American College of Chest Physicians. Statement on cardiopulmonary exercise testing. Am J Respir Crit Care Med 2003; 167: 211-277.

Roca J, Whipp BJ. Clinical exercise testing. Eur Respir Mon 1997; 2: 6.

Wasserman $K$, Hansen JE, Sue $D Y$, Whipp BJ, Casaburi R. Principles of Exercise Testing and Interpretation, 2nd Edn. Philadelphia, Lea \& Febiger, 1994.

Weisman IM, Zeballos RJ. Clinical Exercise Testing. Basel, Karger, 2002.

American Thoracic Society. ATS Statement: Guidelines for the six-minute walking test. Am J Respir Crit Care Med 2002; 166 : 111-117.

\section{References}

1. Wasserman K, Sue DY, Casaburi R, Moricca RB. Selection criteria or exercise training in pulmonary rehabilitation. Eur Respir J 1989; 7: Suppl, 604s-610s.

2. Mathiowetz V, Dove M, Kashman N, Rogers S. Grip and pinch strength: normative data for adults. Arch Phys Med Rehabil 1985; 66: 69-72.

3. McGavin CR, Gupta SP, McHardy GJR. Twelve-minute walking test for assessing disability in chronic bronchitis. BMJ 1976; 1: 822-823.

4. Morgan $A D$, Peck DF, Buchanan DR, McHardy GJR. Effect of attitudes and beliefs on exercise tolerance in chronic bronchitis. BMJ 1983; 286: 171-173.

5. Swinburn $C R$, Wakefield JM, Jones PW. Performance, ventilation, and oxygen consumption in three different types of exercise test in patients with chronic obstructive lung disease. Thorax 198; 40: 581-586.

6. ATS/ACCP Statement on cardiopulmonary exercise testing. Am J Respir Crit Care Med 2003; 167: 211-277.

7. Hermansen L, Saltin B. Oxygen uptake during maximal treadmill and bicycle exercise. J Appl Physiol 1969; 25: 31-37.

8. McArdle WD, Katch FI, Pechar GS. Comparison of continuous and discontinuous treadmill and bicycle tests for max V02. Med Sci Sports Exerc 1973; 5: 156-160.

9. Astrand PO. Saltin B. Maximal oxygen uptake and heart rate in various types of muscular activity. J Appl Physiol 1961; 16: 977-981.

10. Steele $B$, Holt N, Belza B, Ferris S, Lakshminaryan S, Buchner B. Quantitating physical activity in COPD using a triaxial accelerometer. Chest 2000; 117: 1359-1367.

11. Stevens D, Elpern E, Sharma K, Szidon P, Ankin M, Kesten $S$. Comparison of hallway and treadmill sixminute walk tests. Am J Respir Crit Care Med 1999; 160: 1540-1543.

12. Troosters $T$, Vilaro J, Rabinovich $R$, et al. Physiological responses to the 6-min walk test in patients with chronic obstructive pulmonary disease. Eur Respir J 2002; 20: 564-569.

13. Casas A, Vilaro J, Rabinovich $R$, Mayer $A F$, Valera $J L$, Bertoni E. Encouraged six minute walking test reflects 'maximal' sustainable exercise performance in COPD patients. Eur Respir J 2002; 20: 285S.

14. Solway $S$, Brooks D, Lacasse $Y$, Thomas S. A qualitative systematic oveniew of the measurement properties of functional walk tests used in the cardiorespiratory domain. Chest 2001; 119: 256-270.

15. Singh SJ, Morgan MDL, Scott S, Walters D, Hardman $A E$. The development of the shuttle walking test of disability in patients with chronic airways obstruction. Thorax 1992; 47: 1019-1024.

16. Belza B, Steele BG, Hunziker J, Lakshminaryan S, Holt $L$, Buchner DM. Correlates of physical activity in chronic obstructive pulmonary disease. Nurs Res 2001; 50: 195-202.

17. Astrand I. Aerobic work capacity in men and women. Acta Physiol Scand 1960; 49: Suppl. 169, 7-92.

18. Froelicher VF, Brammel H, Davis G, Noguera I, Steward A, Lancaster MC. A comparison of the reproducibility and physiologic response to three maximal treadmill exercise protocols. Chest 1974; 65: 512-517.

19. Froelicher VF, Thompson AJ, Noguera I, Davis G, Stewart AJ, Triebwasser JH. Prediction of maximal oxygen consumption. Comparison of the Bruce and Balke protocols. Chest 1975; 68: 331-336.

20. Debigare $R$, Maltais F, Mallet M, Casaburi R, Leblanc $P$. Influence of work rate incremental rate on the exercise responses in patients with COPD. Med Sci Sports Exerc 2000; 32: 1365-1368.

21. Franklin BA, Gordon S, Timmis GC, O'Neill WW. Is direct physician supervision of exercise stress testing routinely necessary? Chest 1997; 111: 262-265.

22. Wilson RC, Jones PW. Differentiation between the intensity of breathlessness and the distress it evokes in normal subjects during exercise. Clin Sci (Lond) 1991; 80: $65-70$.

23. Cotes JE. Rating respiratory disability: a report on behalf of a working group of the European Society for Clinical Respiratory Physiology. Eur Respir J 1990; 3: 1074-1077.

24. Jones NL, Makrides L, Hitchcock C, Chypchar T, McCartney N. Normal standards for an incremental progressive cycle ergometer test. Am Rev Respir Dis 1985; 131: 700-708.

25. Wasserman K, Hansen JE, Sue DY, Whipp BJ, Casabur $R$. Principles of exercise testing and interpretation. 2nd Edn. Philadelphia, Lea \& Febiger, 1994.

26. Killian KJ, Leblanc P, Martin DH, Summers E, Jones NL, Campbell EJM. Exercise capacity and ventilatory, circulatory, and symptom limitation in patients with chronic airflow limitation. Am Rev Respir Dis 1992; 146: 935-940.

27. Weisman IM, Zeballos RJ, eds. Clinical Exercise Testing. Basel, Karger, 2002.

28. Weisman IM, Zeballos RJ. An integrative approach to the interpretation of cardiopulmonary exercise testing. In: Weisman IM, Zeballos RJ, eds. Clinical Exercise Testing. Basel, Karger, 2002; pp. 300-320.

29. Whipp BJ, Wagner PD, Agusti A. Factors determining the response to exercise in healthy subjects. In: Roca J, Whipp BJ, eds. Clinical Exercise Testing. Eur Respir Mon 1998; 2: 6, 3-31.

30. Roca J, Whipp BJ. Clinical exercise testing with reference to lung diseases: indications, standardization and interpretation strategies. Eur Respir J 1997; 10: 2662-2689.

31. Dempsey JA. Is the lung built for exercise? Med Sci Sports Exer 1986; 2: 143-155.

32. Dekhuijzen PNR, Folgering $H$ Th M, van Herwaarden CLA. Target-flow inspiratory muscle training during pulmonary rehabilitation in patients with COPD. Chest 1991; 99: 128-133.

33. Efthimiou J, Fleming J, Gomes C, Spiro SG. The effect of supplementary oral nutrition in poorly nourished patients with chronic obstructive pulmonary disease. Am Rev Respir Dis 1988; 137: 1075-1082.

34. O'Donnell DE. Exercise limitation and clinical exercise testing in chronic obstructive pulmonary disease. In: Weisman IM, Zeballos RJ, eds. Clinical Exercise Testing. Basel, Karger, 2002: pp. 138-158.

35. Mak VHF, Bugler JR, Roberts CM. Effect of arterial oxygen saturation on six minute walking distance, perceived effort, and perceived breathlessness in patients with airflow limitation. Thorax 1993; 48: 33-38.

36. Wijkstra PJ, Ten Vergert EM, Van der Mark Th W, et al. Relation of lung function, maximal inspiratory pressure, dyspnoea, and quality of life with exercise capacity in patients with chronic obstructive pulmonary disease. Thorax 1994; 49: 468-472.

37. Agusti ACN, Barbera JA, Roca J. Hypoxic pulmonary vasoconstriction and gas exchange during exercise in chronic obstructive pulmonary disease. Chest 1990; 97: 268-275.

38. Gosselink R, Troosters T, Decramer M. Peripheral muscle weakness contributes to exercise limitation in COPD. Am J Respir Crit Care Med 1996; 153: 976-980.

39. Mador MJ, Kufel TJ, Pineda L. Quadriceps fatigue after cycle exercise in patients with chronic obstructive pulmonary disease. Am J Respir Crit Care Med 2000; 161: 447-453. 
40. Saey $D$, Debigare $R$, Leblanc $P$, et al. Contractile leg fatigue after cycle exercise: a factor limiting exercise in patients with chronic obstructive pulmonary disease. Am J Respir Crit Care Med 2003; 168: 425-430.

41. Guyatt GH, Pugsley SO, Sullivan MJ, Thompson PJ, Berman L, Jones NJ. Effect of encouragement on walking test performance. Thorax 1984; 39: 818-822.

42. van Stel HF, Bogaard JM, Rijssenbeek-Nouwens $L H$, Colland VT. Multivariable assessment of the 6-min walking test in patients with chronic obstructive pulmonary disease. Am J Respir Crit Care Med 2001; 163 : 1567-1571.

43. American Thoracic Society. ATS Statement: guidelines for the six-minute walking test. Am J Respir Crit Care Med 2002; 166: 111-117.

44. Sciurba F, Criner GJ, Lee SM, et al. Six-minute walk distance in chronic obstructive pulmonary disease: reproducibility and effect of walking course layout and length. Am J Respir Crit Care Med 2003; 167: 1522-1527.

45. Rabinovich RA, Vilaro J, Roca J. Evaluation exercise tolerance in COPD patients: the 6-minute walking test. Arch Bronconeumol 2004; 40: 80-85.

46. Singh SJ, Morgan MDL, Hardman AE, Rowe C, Bardsley $P A$. Comparison of oxygen uptake during conventional treadmill test and the shuttle walking test in chronic airflow limitation. Eur Respir J 1994; 7: 2016-2020.

47. Revill SM, Morgan MDL, Singh SJ, Williams J, Hardman $A E$. The endurance shuttle walk: a new field test for the assessment of endurance capacity in chronic obstructive pulmonary disease. Thorax 1999; 54: 213-222.

48. O'Donnell DE, McGuire MA, Samis L, Webb KA. General exercise training improves ventilatory and peripheral muscle strength and endurance in chronic airflow limitation. Am J Respir Crit Care Med 1998; 157: 1489-1497.

49. O'Donnell DE, Webb KA. Measurement of symptoms, lung hyperinflation, and endurance during exercise in chronic obstructive pulmonary disease. Am J Respir Crit Care Med 1998; 158: 1557-1565.

50. van 't Hul AJ, Gosselink R, Kwakkel G. Constant-load cycle endurance performance: test-retest reliability and validity in patients with COPD. J Cardiopulm Rehabil 2003; 23: 143-150.

51. Neder JA, Jones PW, Nery LE, Whipp BJ. Determinants of exercise endurance capacity in patients with chronic obstructive pulmonary disease. The power-duration relationship. Am J Respir Crit Care Med 2000; 162 : 497-504.

52. Enright $P L$, Sherrill $D L$. Reference equations for the sixminute walk in healthy adults. Am J Respir Crit Care Med 1998; 158: 1384-1387.

53. Troosters $T$, Gosselink R, Decramer M. Six minute walking distance in healthy elderly subjects. Eur Respir J 1999; 14: 270-274.

54. Redelmeier DA, Bayoumi AM, Goldstein RS, Guyatt GH. Interpreting small differences in functional status: the six minute walk test in chronic lung disease patients. Am J Respir Crit Care Med 1997; 155: 1278-1282.

\section{Suggested answers}

1. The main indications for exercise tesing are: impaired exercise tolerance; to find out which system is limiting exercise; to establish which organ systems have an abnormal response during or after exercise; to investigate how much exercise is appropriate/safe in a patient; and to determine the response to treatment.

2. Incremental exercise tesing is used to assess impaired exercise capacity, to investigate factors limiting exercise performance or to prescribe exercise training. Field tests are used to investigate the effects of interventions that alter submaximal or endurance exercise capacity.

3. Differences in work load increment size result in changes in maximal work load: larger increments=higher peak work load.

4. See table 3 for full guidelines.

5. Ventilatory limitation is caused by an imbalance between the workload and the capacity of the respiratory muscles. See table 2 for more details.

6. The changes in oxygen saturation, $\mathrm{VO}_{2}, \mathrm{HR}$ and hypercapnia observed during $6 \mathrm{MWT}$ are close to those obtained during incremental exercise tests, and, therefore, investigators should look out for alarm symptoms that may occur.

7. Yes, in patients with less severe airway obstruction ( $\mathrm{FEV}_{1}>50 \%$ predicted).

8. No, field tests are the test of choice for the longtitudinal assessment of exercise performance, e.g. when assessing the effects of pulmonary rehabiliation.

9. Yes, especially in patients with significant complaints of leg fatigue at the end of the exercice test.

10. Exercise laboratories should be encouraged to test some healthy control subjects in the age span of interest to evaluate which normal values best suit their laboratory setting.

\section{Suggested websites}

www.acc.org/clinical/guidelines/ exercise/dirindex.htm American College of Cardiology Foundation. ACC/AHA 2002 Guideline Update for Exercise Testing.

www.americanheart.org American Heart Association. Guidelines for Clinical Exercise Testing Laboratories.

www.lww.com/product/_0-68330355-4

American College Sports Medicine. Guidelines for exercise testing and prescription.

www.guideline.gov/summary/su mmary.aspx_doc_id=2844 National Guideline Clearinghouse: exercise testing for evaluation of hypoxemia and/or desaturation.

www.guideline.gov/summary/su mmary.aspx_doc_id $=3427$

American College of Cardiology/American Heart Association Task Force on Practice Guidelines. 